MODELING, IDENTIFICATION AND CONTROL, 2003, vOL. 24, No. 3, 159-167

doi:10.4173/mic.2003.3.2

\title{
A Global Remote Laboratory Experimentation Network and the Experiment Service Provider Business Model
}

\author{
TOR IVAR EIKAAS ${ }^{*}$, CHRISTIAN SCHMID $¥$, BJARNE A. FOSS§ \\ and DENIS GILLETy
}

Keywords: Remote experimentation, education, training, eBusiness

\begin{abstract}
This paper presents results from the IST KAII Trial project ReLAX-Remote LAboratory eXperimentation trial (IST 1999-20827), and contributes with a framework for a global remote laboratory experimentation network supported by a new business model. The paper presents this new Experiment Service Provider business model that aims at bringing physical experimentation back into the learning arena, where remotely operable laboratory experiments used in advanced education and training schemes are made available to a global education and training market in industry and academia.

The business model is based on an approach where individual experiment owners offer remote access to their high-quality laboratory facilities to users around the world. The usage can be for research, education, on-the-job training etc. The access to these facilities is offered via an independent operating company - the Experiment Service Provider. The Experiment Service Provider offers eCommerce services like booking, access control, invoicing, dispute resolution, quality control, customer evaluation services and a unified Lab Portal.
\end{abstract}

\section{Introduction}

The Global Remote Laboratory Experimentation Network and the Experiment Service Provider Business Model is based on the idea that although a physical process cannot easily be moved, it can be made available for on-line remote experimentation via the Internet. The providers (owners) of laboratory facilities will be paid for the use of their facilities, while the customers are relieved of the costs involved in building and maintaining the laboratory equipment. This also enables a much more efficient use of laboratory facilities, since laboratory equipment in universities is at present used for only a fraction of the academic year. A key aspect addressed in this paper is the assumption that a commercial company, the Experiment Service Provider, is needed for providing the dedicated services required by this new remote experimentation paradigm. As a matter of fact, classical universities are not organised adequately to offer such Internet services.

The remote experimentation providers and users, as well as the Experiment Service Provider, together form a network of partners. During a 24-month period from late 2001, a trial network obtains funding from the European Commission

Presented at the eBusiness and eWork Conference (2002), 16-18 October 2002, Prague, the Czech Republic.

$\dagger$ Cyberlab.Org AS, PO Box 2853 Elgseter, N-7465 Trondheim, Norway. Tel: +4792604 524; Fax: +47 735943 99; Email: Tor.I.Eikaas@cyberlab.org

\$Ruhr-Universiät Bochum, Germany.

$\S$ Norwegian University of Science and Technology, Trondheim, Norway.

ๆ Swiss Federal Institute of Technology, Lausanne, Switzerland.

*Author to whom correspondence should be addressed. 
through the IST programme to conduct the ReLAX 'Remote LAboratory eXperimentation trial' project (ref. ReLAX), which includes participants from different countries. The participants of the ReLAX project are from Norway (Cyberlab.Org AS, Prediktor AS, Norwegian University of Science and Technology (NTNU)), Germany (Ruhr-Universität Bochum (RUB)) and from Switzerland (Swiss Federal Institute of Technology (EPFL)).

\section{Background}

During the last decades there has been a decline in the use of physical experiments in engineering education. Learning is accomplished through a diversified set of activities like lectures, paper and pencil exercises, simulators, pilot experiments, discussions, and report writing. It is widely recognised that a mixture of theoretical and practical exercises is vital for efficient learning, and physical experiments constitute an important and complementary ingredient in learning, especially in the engineering and physics domain (Antsaklis et al., 1999). Engineering students need to put the theory they learn during lectures into practice, which includes experimentation on real processes. Even quite rigorous simulators do not fully demonstrate the many practical problems related to operation of real-world equipment, and the trend towards the increased use of simulators in learning programs can only partially compensate for the decline in the use of physical experiments. There are several reasons for the decline described above. One important reason is the fact that physical experiments are costly to develop, maintain and operate. Another reason has been a strong belief that simulators can fully replace physical experiments. The ability to simulate a process is clearly helpful for finding solutions to real-world problems. Nevertheless, it is the strong believe of the authors that physical experiments constitute an important ingredient to real hands-on learning activities.

\section{Cyberlab and the Experiment Service Provider solution}

The main objective of ReLAX is the trial of a new business model based on an Experiment Service Provider (ESP) offering services utilising remote experimentation.

The idea behind the business model is to organise the network of laboratory experiments through an independent organising company, the Experiment Service Provider. The ESP offers a set of laboratory experiments to Customers (universities, individuals and industrial companies) on a global basis through a lab-portal and accompanying eCommerce services, see Figure 1 . The experiments are high quality experiments offered by educational institutions (Providers).

The ESP is responsible for the commercial aspects like quality assurance/quality control, marketing, booking system for assigning experiments and time slots, access control, invoicing, secure electronic payment, handling of disputes, SW services, realtime monitoring of labs, etc.

A new company Cyberlab (Cyberlab) has been set up with the main objective to act as the Experiment Service Provider in this network. The Cyberlab-company is independent of other companies offering SW and HW for remote operation, and a main objective is to provide easy integration in the network of any HW and SW system used by the Provider.

The Provider will connect a laboratory experiment to the Internet through a Web service, and the user interacts with the experiment through a Web browser. The 


\section{Experiment Service Provider Independent operating Company}

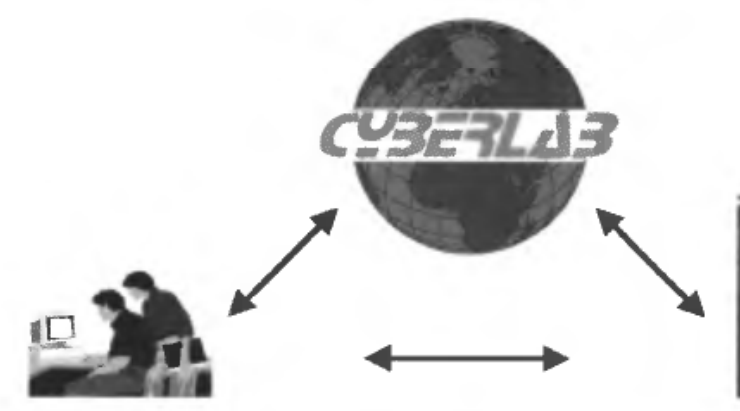

Customers

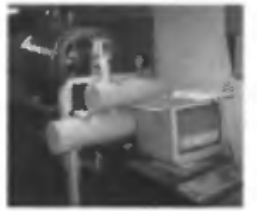

Providers
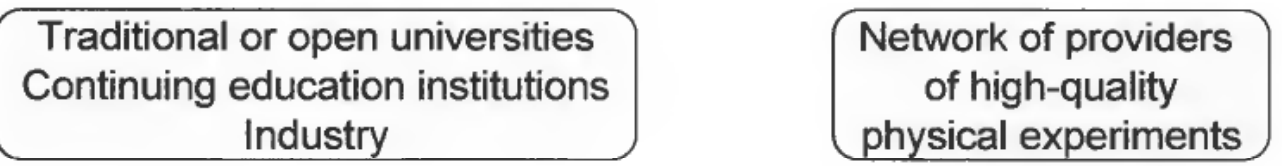

Figure 1. Organisation of the remote laboratory experimentation network.

information received from the experiment will typically include time series plots, video and audio information. When the experiment is completed, it should also be possible to obtain comprehensive documentation of the experiment by downloading files consisting of time series of experimental results. Exactly which variables the user should be able to manipulate remotely, as well as when, how and how much the variables can be manipulated, will depend on the experimental design.

The Experiment Service Provider will specify key aspects of how the user should be able to interact with the process, and will assist the Provider in connecting the experiment to the Internet, if the Provider requires such assistance. However, technical specifications will be independent of the actual hardware and software the Provider uses.

Each Provider may concentrate their effort on maintaining a few high-quality experiments for remote on-line experimentation, while having access to many other remote laboratories from other Providers. Further, other users, without their own laboratory facilities, may purchase access to remote laboratories. This latter category of users will include universities that have given up on practical experiments due to high costs, open universities, commercial organisations offering continuing education courses, and in-house training departments in engineering and production companies. There are a number of technological and practical issues that need to be resolved in order to achieve some kind of closeness to the same learning from running a remote experiment as from running the same experiment locally.

Within the Cyberlab network, an external advisory board is being established. The objective of this group is to obtain the right interaction with possible future customers from industry and academia including open learning institutions. In addition, the advisory board will evaluate the quality, suitability, etc. of new labs for inclusion in the network. The world leading traditional universities, distributors of distance education and industrial companies are invited to participate in the network. 


\subsection{Economic benefit}

The main assumptions on the economic benefits by joining the network have been reinforced and clarified during the ReLAX project. By joining this network, the experiment Providers can benefit from the services from the ESP and at the same time benefit from an increased income through the exploitation of own laboratory resources. The underlying idea of the business model is to establish a win-win situation where all involved parties profit from their participation:

- The Experiment Service Provider by charging a certain percentage of the total Customer cost for running an experiment and/or from membership fees.

- The Providers by generating income from the remote operation of their local laboratory infrastructure.

- The Customers by avoiding large investments in laboratory development, operation and maintenance costs and by only paying for the efficient use of selected high-quality relevant remote experiments. The educational benefit from the Customers is first of all through the access to high-quality experiments bringing added value for own education, training or services.

A world-wide survey conducted by Cyberlab (Balchen \& Eikaas, 1999, 2000) and the ReLAX project confirm the underlying assumption of the business model for the university market segment:

- The situation for many educational institutions is that they have problems with maintenance and operation of these complex laboratories due to the high costs.

- The labs are only used locally a fraction of the available time.

- The Cyberlab initiative is regarded as a very timely initiative making it possible to generate sufficient income that can justify the maintenance and operational costs.

Several possible payment models have been evaluated and investigated during the ReLAX project. The results so far, indicate that the network must support both lowcost/free labs as well as the high-cost ones. This means that several payment models will co-exist in the Cyberlab system, but the main principle is as follows:

- The Providers pays a certain membership fee for getting included in the Cyberlab Lab portal.

- Only a limited set of the ESP services will be available in connection with the free labs.

- The cost for running the other labs is based on an evaluation of 'current market price' for the experiment, and is subject to an agreement between the ESP and the Provider.

- The ESP receives an agreed percentage of the cost for running an experiment.

The ESP system implemented by Cyberlab offers a flexible solution with options for flexible framework agreements, unlimited access during a specified period of time, differentiation of the pricing depending on customer category and usage.

\section{Deployment of Remote Experiments}

The main concept in turning a locally-controlled experiment into a remotelycontrolled one consists of moving the user interface of the monitoring and control 
software away from the physical system. Such a paradigm enables a flexible access that is valuable for teachers looking for live classroom demonstration facilities or for students wishing or having to carry out real-world laboratory projects to sustain their learning activities.

In automatic control education, different experiments have to support different concepts taught in control courses like controller tuning, model development, and the actual programming and implementation of a control scheme or algorithm. A rich variety of control experiments can be carried out remotely in the Cyberlab framework. Three different laboratory experiments are initially available through the ReLAX project; one at EPFL, NTNU and RUB, respectively. Some typical examples from these experiments are given in the following sections. A more detailed description of the labs is given in Schmid et al. (2001).

\subsection{Experiments where the user acts as an operator for the laboratory process}

Typically, the user will change the reference values of some control loops and monitor the responses from the equipment. Such parameter changes may be based on intuition only, or result from a systematic tuning procedure, which may be supported in the experimentation environment. The experiment may also be designed to introduce disturbances in order to validate the dynamic performances obtained with the user's settings. In Figure 2, an example of a user interface for making these kinds of experiments with an inverted pendulum is shown (Gillet et al, 2000).

This pendulum emulates in two dimensions a juggler trying to keep a broomstick in equilibrium on his fingertip. The inverted pendulum is made up of a 4-meter horizontal rail mounted on top of a case. A cart, moved by an electric motor by means of a metallic belt, can slide along this rail. The control objective is to simultaneously keep the pendulum stick in the upright position and the supporting cart at the center of the rail, using a single actuator.

Any type of computer equipped with a National Instruments PCI-1200 family DAQ board can control this laboratory-scale system. In the actual implementation, the computer is an Apple Power Macintosh G3 which handling simultaneously the real-time control, the video processing and broadcasting, the transactions with the service provider, as well as the data exchange with remote users. The control SW

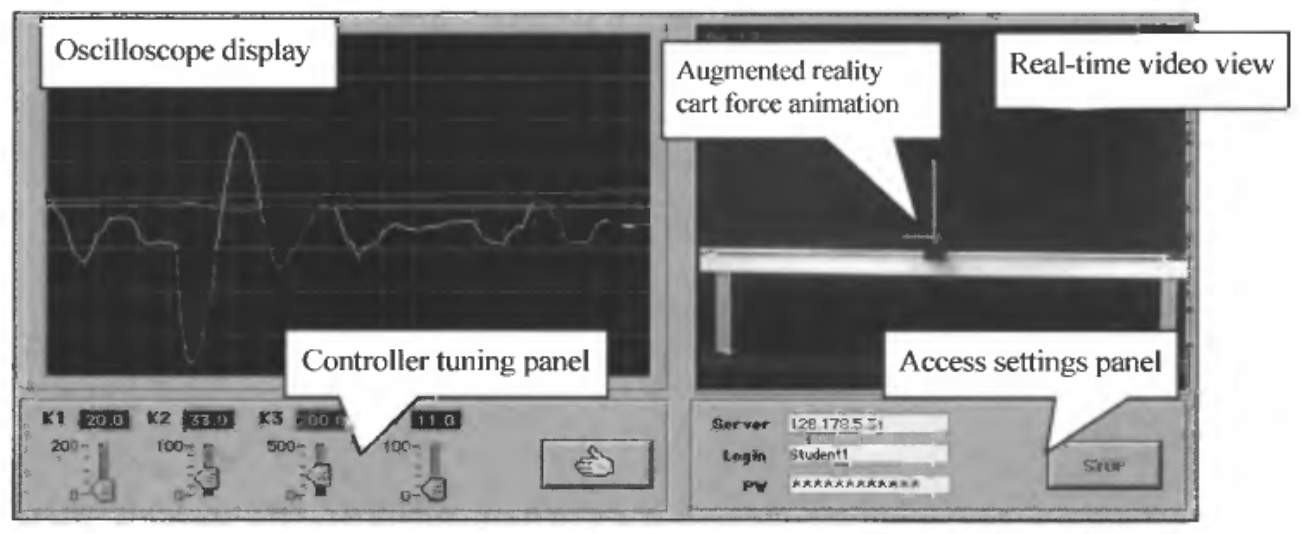

Figure 2. Sereenshot of the inverted pendulum client software. 
piloting the real process locally is built using LabVIEW. A real-time kernel extends the LabVIEW capability.

\subsection{Experiments in which the user may learn about industrial control systems, including instrumentation and actuators}

Learning about industrial control systems, including instrumentation and actuators, is performed by activating hyperlinks on a Process and Instrument Diagram of the Remote Laboratory. A link may e.g. point to a datasheet for a valve. The refrigeration process located at NTNU is suited for this since it contains a number of different instruments, valves and process elements. This process itself consists of a compressor, condenser, expansion valve, and an evaporator, as shown schematically on the left-hand side of Figure 4. The process will cool down the liquid in a heated tank. The process is equipped with approximately 30 temperature, pressure, level and flow transmitters, and 10 valves. The instrumentation is connected to a Simatic S7-400 PLC, which again is connected to an industry standard PC. This PC has software for communicating with the PLC and for programming the PLC. In addition, Simatic WinCC is installed on the PC, and is used both for a local HMI and for communicating with a Web server through an OPC interface. The Web server uses a program suite from Prediktor AS to access process data from WinCC and make this data available over the Web.

The use of Web technology also allows for a tight integration of tutorial material on the process or the control theory, which the experiments are designed to demonstrate. This is illustrated in Figure 4, see also Balchen et al. $(1999,2000)$. By pointing to key equipment in the process, a pressure-enthalpy diagram pops up on the righthand side, in which a refrigeration cycle is drawn in. It also explains where the equipment pointed to is located in the pressure-enthalpy diagram.

The user may run different experiments on the refrigeration cycle, e.g.: (a) Identification experiments by perturbing the system in order to identify an empirical dynamical model, to determine parameters in model, or to validate a model. (b) Testing of different control structures. (c) The user may program a controller and download it to the control system, and then assess its performance by running experiments with this controller.

\subsection{Experiments with feeling of being present in the laboratory}

One important issue that needs to be addressed in remote experimentation is how to give the user the necessary feeling of interacting with real, physical equipment. The ability to manipulate the experiment remotely, and seeing the results of such manipulations in real time, will contribute to this aim, see Figure 2. The feeling of being present at the experimental site can be further enhanced by the transmission of sound and video images from the experiment, and by the use of virtual-reality techniques. Figure 4 shows an example from an optical tracking plant (Junge \& Schmid, 2000), where the experiment can be optionally controlled by a virtual-reality (VR) model and the reaction of the plant can be immediately observed by the video and audio connection.

The plant is composed of two units. The reference unit includes a light source fixed on a wheel. A motor moving the light spot on a circular trajectory drives this wheel. The user can select the angle (i.e. position of the light spot) or the angular 


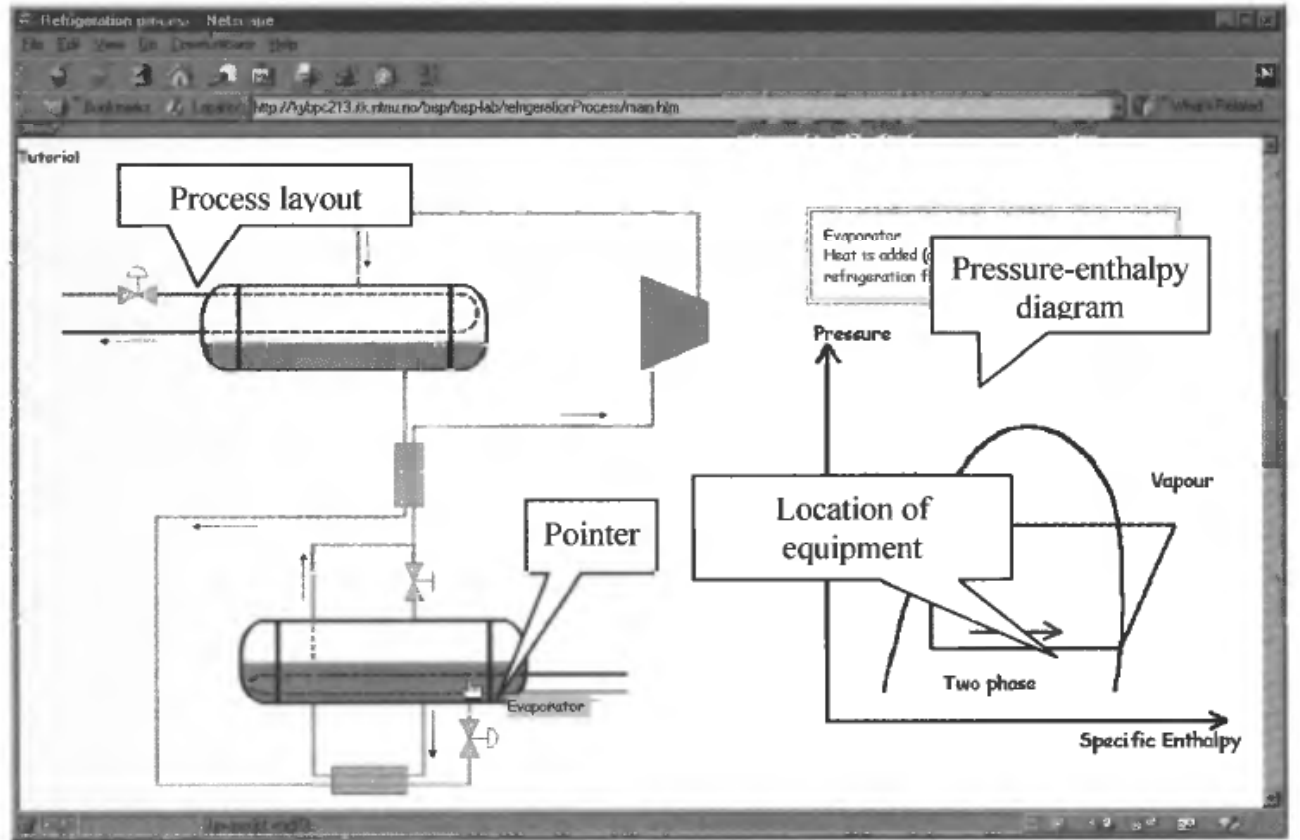

Figure 3. Tutorial material on the process is integrated with drawings of the process layout.

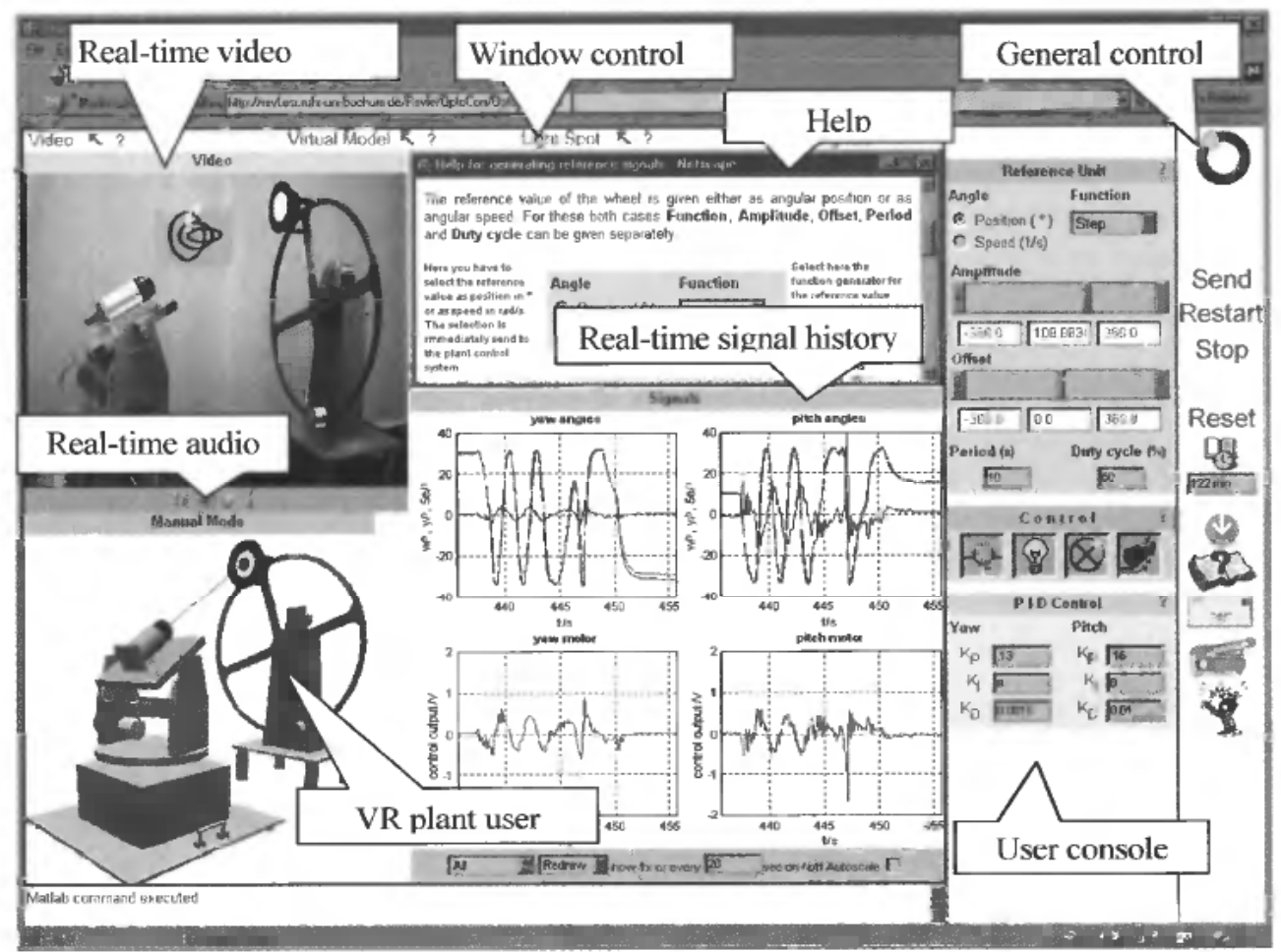

Figure 4. Screenshot example of the GUI for the optical tracking plant with virtual-reality plant user interface. 
velocity of the wheel (i.e. speed of the light spot). The challenge for the users is to design a controller such that the light spot will not leave the camera's view. Further details are described in Schmid (2001).

The Web page necessary to control this experiment is organised in the form of a cockpit. The main operating actions are located in the general control panel frame. The specific commands to operate the plant as well as parameters to modify the characteristics of the reference signal and of the implemented controller are found in the user console panel. The lack of a direct physical contact with the experiment is compensated by enhanced multimedia components. A $V R$ plant user interface enables the user to operate the real plant manually in the virtual reality scene of the 3-D model. The actions performed with this model are immediately converted into commands and transferred to the experimental site.

\section{Experiences from the trial project}

The educational benefits of utilising a remote experimentation network as described in this paper have been evaluated in the ReLAX project. Students in different countries have run the three experiments described above as part of both regular courses and special targeted test sessions. The Cyberlab network has also been used successfully outside the ReLAX project. Some of the labs have been used by students from the US in special training sessions. In addition, the Refrigeration cycle lab at NTNU has been used in industrial training courses focussed on training needs for process operators from different industrial segments.

The main conclusion is that carefully designed laboratory experiments together with well designed user interfaces results in solutions where the educational benefits are comparable with local operation of the equipment. An interesting finding is that female students in general are more in favour of remote experimentation than the male students are.

The business benefits have been evaluated in the ReLAX project. The main conclusion is that the ESP services offered by Cyberlab are sufficient both with respect to quality and functionality in order to operate the network, and second, the results clearly show that there is a potential market for this type of services based on the proposed business model. Some obstacles for sustainable business activities have however been identified and confirmed. The main obstacles are:

- The Customers' willingness to buy access to laboratory resources. This is primarily valid for academic Customers, and not for the industrial Customers.

- The availability of a sufficient number of labs well suitable for integration in complete educational packages.

- The robustness and technical quality for available experiments.

The next steps after the ReLAX project will focus on:

- Building the global network with sufficient number of laboratories well suited for and ready to be integrated in complete educational packages, both for the industry and the academia.

- Exploit the Experiment Service Provider business model with the objective to establish sustainable business activity based on these services.

The main exploitation of the business model is done by Cyberlab after the end of the ReLAX project. Other projects world-wide have already suggested to use Cyberlab and the Experiment Service Provider business model in their remote experimentation activities. 


\section{Conclusions}

This paper describes a model for establishing a sustainable global laboratory experimentation network supported by the Experiment Service Provider business model. The benefits of the network and the business model are demonstrated via a trial setup using three labs in different countries together with Cyberlab as the Experiment Service Provider. The economic benefits for all actors involved have been clarified during the project, but more market development must be done before sustainable business activities can be achieved.

\section{Acknowledgements}

The authors gratefully acknowledge the support received from the European Commission under contract IST-1999-20827.

\section{References}

Antsaklis, P., Basar, T., De Carlo, R., McClamroch, N. H., Spong, M. and Yurkovich, S. (1999). Report on the NSF/CSS Workshop on New Directions in Control Engineering Education. IEEE Control Systems Magazine, 19, No. 5, pp. 53-58.

ReLAX--Remote LAboratory eXperimentation trial. IST project IST-1999-20827. Web page http://www.relax-project.com

BALChen, J. G and EIKAAS, T. I (1999, 2000). Results from a world-wide questionnaire on remote experimentation, Internal report, Cyberlab.

Cyberlab-The Experiment Service Provider and Laboratory Portal. Web page http:// www.cyberlab.org

Foss, B. A., EIKAas, T. I. and Hovd, M. (2000). Merging physical experiments back to the learning arena. Proc. American Control Conference ACC'2000, Chicago, pp. 2944-2948.

Gillet, D., Salzmann, C., Latchman, H. A. and Crisalle, O. D. (2000). Recent Advances in Remote Experimentation. Proc. American Control Conference ACC'2000, Chicago, pp. 2955-2956.

Junge, Th. F. and Schmid, Chr. (2000). Web-Based Remote Experimentation Using a Laboratory-Scale Optical Tracker. Proc. American Control Conference ACC'2000, Chicago, pp. 2951-2954.

Kheir, N. A., Áström, K. J., Auslander, K. C., Cheok, D., Franklin, G. F., Masten, M. and Rabins, M. (1996). Control Systems Engineering Education. Automatica, 32, No. 2, pp. 147-166.

SCHMID, Chr. (2001). Web-Based Remote Experimentation. Proc IFAC Conference on Telematics Applications in Automation and Robotics, Weingarten.

Schmid, C., EikaAs, T. I., Foss, B. and Gillet, D. (2001) Remote Laboratory Experimentation Network, TA2001, Weingarten, Germany. 